\title{
Factor Structure of Zimbardo Time Perspective Inventory (ZTPI) in a Norwegian Convenience Sample
}

\author{
Jens Christoffer Skogen ${ }^{1,2,3, *}$ and Sverre Nesvåg ${ }^{2,3}$ \\ ${ }^{1}$ Department of Health Promotion, Norwegian Institute of Public Health, Bergen, Norway \\ ${ }^{2}$ Centre for Alcohol and Drug Research, Stavanger University Hospital, Stavanger, Norway \\ ${ }^{3}$ Faculty of Health Sciences, University of Stavanger, Stavanger, Norway
}

Received 11 January 2019; accepted 10 April 2019

\begin{abstract}
Sense of time is a fundamental aspect of human psychology. The Zimbardo Time Perspective Inventory (ZTPI) is a widely used questionnaire meant to measure fundamental experiential dimensions of time, such as past, present and future. The aim of this study was to establish model fit of a Norwegian extended version of the ZTPI. The study is based on a convenience sample of 713 individuals. Based on previous findings, we employed confirmatory factor analysis and exploratory structural equation modelling to investigate different factor structures of ZTPI. The analyses were carried out using the WLSMV-estimation approach, and several fit indices was used as indicators of how well the data fitted the suggested factor structure. This first investigation of a Norwegian version of ZTPI did not find support for the original 56 -item scale, the S-ZTPI version ( 64 items), nor an extended version that also incorporated the transcendental time perspective (74 items). In post-hoc analyses, we identified a model with 34 items and 7 factors that fitted the data adequately. Further studies should investigate the factor structure of ZTPI in a Norwegian context, and international studies should investigate how the transcendental time perspective relates to the rest of ZTPI.
\end{abstract}

Keywords

Time perspective, temporal orientation, confirmatory factor analysis, reliability, ZTPI

\section{Introduction}

Sense of time is a fundamental part of human psychology (Di Lernia et al., 2018), and has been recognized as such for a long time. Recently, however, sense of time and individual's time perspective have been given further importance and become a growing area of scientific endeavour. Time perspective can be understood

* To whom correspondence should be addressed. E-mail: jens.christoffer.skogen@fhi.no 
as a fundamental cognitive dimension dividing human experience into past, present and future (Zimbardo \& Boyd, 1999). Time perspective has, for instance, been used to investigate differences in risk taking, health-promoting behaviour, quality of life, aging and as a predictor of drug use (Boyd \& Zimbardo, 2005; Hamilton et al., 2003; Keough et al., 1999; Loose et al., 2018; Preau et al., 2007; Rohr et al., 2017; Stolarski et al., 2018; Zimbardo et al., 1997).

One way to measure time perspective is the Zimbardo Time Perspective Inventory (ZTPI). ZTPI was presented as a 56-item inventory to measure different aspects of an individual's time perspective, covering past, present and future time perspectives (Zimbardo \& Boyd, 1999). Currently, ZTPI is widely used internationally for different purposes in scientific fields such as psychology, behavioural economics and sociology (Stolarski et al., 2015). Translated versions of ZTPI have been investigated in relation to its psychometric properties and validity in many different countries and settings (Akirmak, 2019; Chan et al., 2016; Sircova et al., 2014; Sobol-Kwapinska et al., 2016; Stolarski et al., 2015). Sircova and colleagues (2014) investigated the structural equivalence across 24 countries and concluded that the ZTPI is invariant across most of the countries included, despite diverse cultural traditions. However, a recent study comparing ZTPI across United States, Poland and Nigeria found differences in model fit across these countries (SobolKwapinska et al., 2018). The authors conclude that there may be cultural differences in time perspectives that need to be considered in future revisions of ZTPI.

The original ZTPI has been investigated as a one-, three- and five-factor inventory using confirmatory factor analyses (CFA; Carelli et al., 2011), but is usually understood as measuring five distinct time perspectives: past negative, past positive, present hedonistic, present fatalistic and future (Sobol-Kwapinska et al., 2018) (Table 1). ZTPI has been subject to many investigations into its factor structure, as well as many modifications to the original inventory. A Swedish version (S-ZTPI) was developed which included eight additional items, and this version was suggested to contain six distinct factors, as the future time perspective was divided into a positive and a negative dimension (Carelli et al., 2011). Zimbardo and colleagues have also suggested a transcendental time perspective questionnaire consisting of 10 items to complement the original ZTPI questionnaire without being incorporated into the scale itself (Boyd \& Zimbardo, 1997). See Table 1 for a short description of the different time-perspectives.

Many previous studies have reported a less than optimal fit of ZTPI (SobolKwapinska et al., 2018). It has been therefore been suggested that further investigations of the factor structure of ZTPI should not rely solely on CFA, but also explore other statistical techniques. Exploratory structural equation modelling (ESEM) has been employed as an alternative and complementary approach with promising results (Sobol-Kwapinska et al., 2018; Temple et al., 2017). ESEM can be especially suitable for complex models with many items and factors that are conceptually closely related (Worrell et al., 2018). The ESEM approach can be seen 


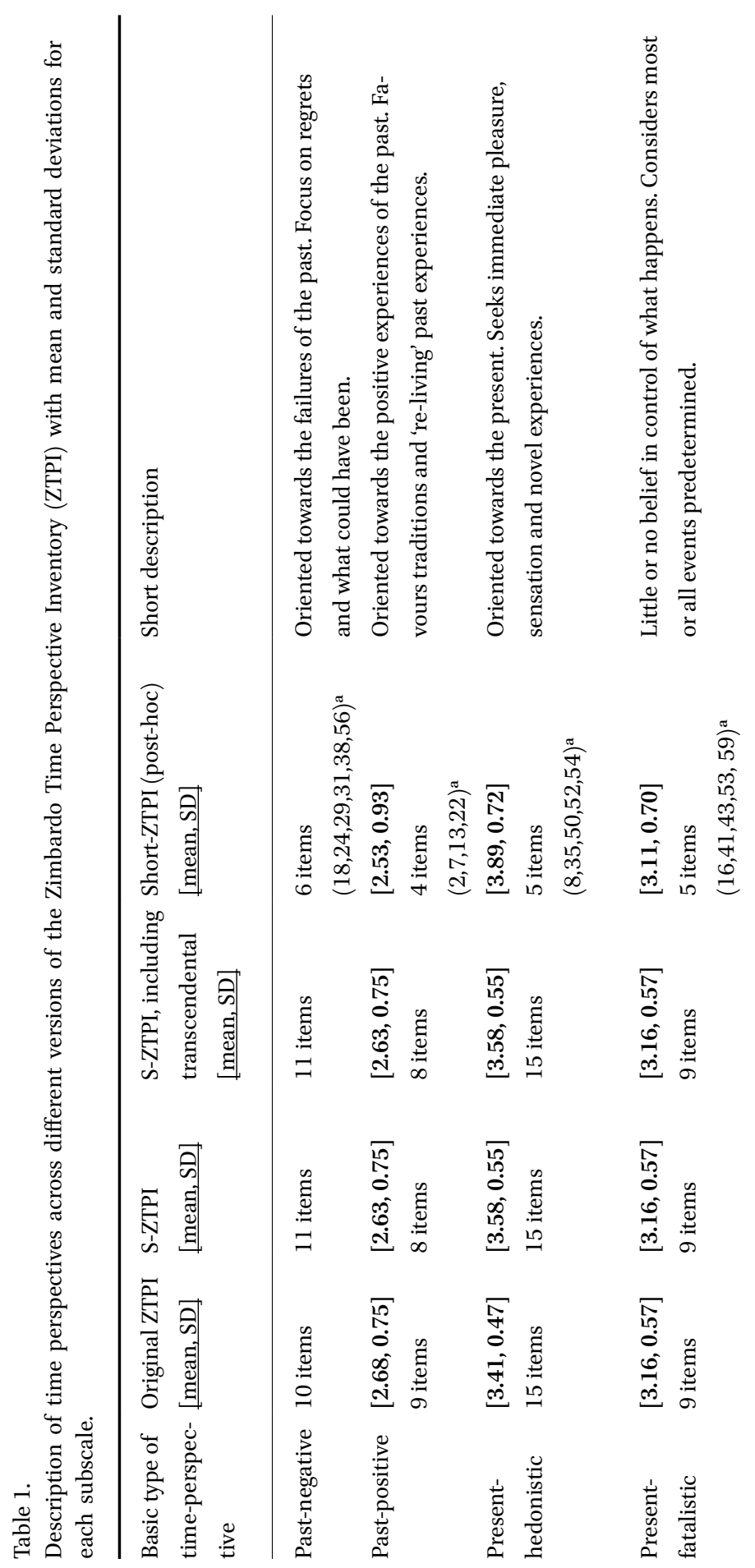




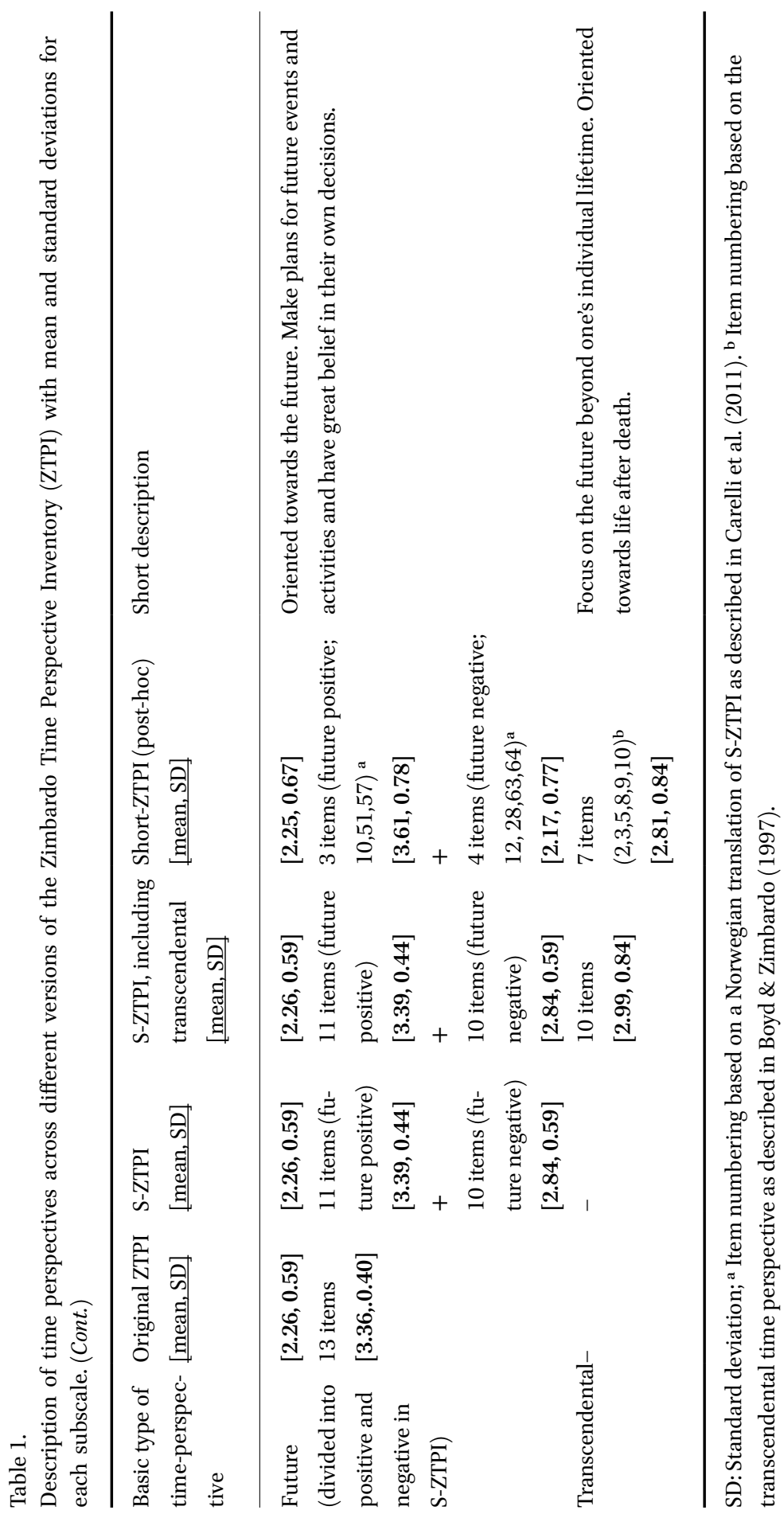


as a combination of exploratory and confirmatory factor analysis by negating the necessity for items to load only on one factor and allowing for non-consequential cross-loadings (Asparouhov \& Muthén, 2009; Marsh et al., 2014).

To the best of our knowledge, a Norwegian version of the full ZTPI has not been investigated in relation to factor structure. Given the potential importance of this inventory in many different areas, it is desirable to pilot the full ZTPI in a Norwegian setting. The present paper does not attempt to cover all of the different versions of ZTPI or cover all of the aspects related to ZTPI's psychometric properties and potential validity. Rather this paper constitutes a first attempt to determine the factor structure of a Norwegian version of ZTPI, with an initial focus on full versions and not short versions of the inventory.

\subsection{Objectives of the Present Study}

The aim of the present study was to compare three different factor structures of ZTPI in a Norwegian sample: The original 56-item scale was investigated, the S-ZTPI version, and an extended version also incorporating the transcendental time perspective.

\section{Material and Methods}

\subsection{Participants}

Participation in the present study was based on convenience sampling of individuals 18 years old or above. An invitation to participate in the study was sent to all 7,800 employees in a general hospital and employees of a larger food-store, via the e-mail systems of the two organizations. Out of the 7,800 individuals invited, $713(9.1 \%)$ participated. The mean age was 33.9 (standard deviation 12.8, range $18-69$ years $)$ and $74.3 \%(n=530)$ were female.

\subsection{Measures}

The English version of ZTPI, including the eight additional items from S-ZTPI (64 items), and the Transcendental-future Time Perspective Inventory (TTPI; 10 items) were translated into Norwegian by two bilingual speakers; one native Norwegian and one native English speaker. Based on a review of the research literature, the native Norwegian speaker had some prior knowledge of ZTPI. The translation consisted of the following steps: First, the bilingual native Norwegian speaker translated the items from English to Norwegian. Then the native bilingual English speaker translated the items back to English. The two translators then compared the back-translated items with the original English version. Only some minor differences were found, and the two translators agreed upon the Norwegian words and syntax that best covered the original English version. 


\subsection{Data Collection}

Data were collected online, and included only self-reported age and gender, as well as the 64-item version of the ZTPI, and TTPI. All of the items were rated on a five-point scale ("very untrue" to "very true"). Skewness ranged from 1.34 to -1.28 and kurtosis from 5.04 to 1.78 across items.

\subsection{Ethical Considerations}

Information about the purpose and procedure of the study was provided in the invitation e-mail. By opening the link to the online survey, all of the participants gave their digital consent to participate in the survey. The content of the questionnaire did not constitute health-related research according to the Norwegian Health Research Act. As such, ethical approval was not required according to Stavanger University Hospital's guidelines and national regulations.

\subsection{Main Analyses: Procedure}

Based on previous findings, we initially employed CFA and ESEM to compare different factor structures of ZTPI. The original 56-item version was analysed as a five-factor structure, and the extended S-ZTPI as a six-factor structure. Finally, an extended version also incorporating the transcendental time-perspective was investigated as a seven-factor structure (74 items). The CFA and the ESEM analyses were carried out using a mean- and variance-adjusted weighted least squares (WLSMV) estimation approach suitable for ordinally scaled items (Asparouhov \& Muthén, 2009; Marsh et al., 2014). Root Mean Square Error of Approximation (RMSEA), Comparative Fit Index (CFI), Tucker-Lewis Index (TLI) and Standardized Root Mean Square Residual (SRMR) were used as indicators of how well the data fitted the suggested factor structure. Several criteria for evaluating model fit have been suggested (Byrne, 2012; Hu \& Bentler, 1999). In the present study, a RMSEA of $<0.08$ is judged as acceptable, and a value of $<0.05$ as good. For CFI and TLI, a value of $>0.90$ is considered acceptable fit, and $>0.95$ good fit. For SRMR, a value $<0.08$ is considered acceptable, while a value of $<0.05$ is considered good fit. Out of the 713 participants, $636(89.2 \%)$ answered all of the 74 ZTPI-questions. Bivariate comparisons of mean response patterns between those answering some of the items versus those with complete data did not reveal any statistically significant differences ( $p$-values ranging from 0.138 to 0.492 ). Full information maximum likelihood (FIML) was therefore used to handle missing data under the assumption that data was missing completely at random (MCAR) or missing at random (MAR) (Enders, 2010). For the factor structure yielding the best fitting model according to the fit indices, Cronbach's alpha was estimated. Configural and scalar invariance across gender and age categories (0-29/30+ years) was also investigated. A decrease in model fit was considered indicative of noninvariance if the decrease was more than 0.015 for RMSEA and more than -0.01 for CFI (Putnick \& Bornstein, 2016). Analyses were performed using R (R Core Team, 
2018) and CFA was performed using the lavaan-package (Rosseel, 2012), while ESEM was performed using Mplus version 8.2 (Muthén \& Muthén, 1998-2017).

\subsection{Post-Hoc Exploratory Analyses}

Considering the results from the main analyses, a post-hoc analysis was conducted in an attempt to improve model fit:

1) Exploratory factor analysis of each of the seven factors independently to verify that the suggested factors were unidimensional and that factor loadings were reasonably high. Items with factor loadings $<0.50$ were removed from further analysis (Hair et al., 1998).

2) ESEM analysis of a seven-factor model using the retained items. Low factor loadings $(<0.50)$ and salient cross-loading items $(>0.30$ on multiple factors) (Rosellini \& Brown, 2011) were removed from further analysis.

3) CFA of each of the seven factors independently using the retained items to verify unidimensionality.

4) CFA and ESEM analysis of a seven-factor model using the retained items.

\section{Results}

\subsection{Main Analyses: CFA and ESEM}

Table 2 gives an overview of the CFA and ESEM fit indices for the original ZTPI, the extended S-ZTPI and the extended version incorporating the transcendental time perspective. For CFA, none of the tested models fitted the data (Table 2). The extended seven-factor model had the least inadequate fit indices, with a RMSEA $=0.053($ CI90\% 0.051-0.054, $p$-value $<0.001)$, CFI $=0.844$, TLI $=0.838$ and SRMR $=0.092$. The correlation between the different factors of the best-fitting model ranged between 0.75 (past negative and future negative; $p<0.001$ ) and -0.02 (future positive and transcendental; $p=0.653$ ) with a mean correlation of 0.34 between remaining factors (all $p$-values $<0.05$ ). The internal reliability of the factors ranged from 0.54 (future positive) to 0.88 (past negative). All factors had one or more item with a loading less $<0.50$, and all except the "transcendental" and "past positive" factor had at least one item with a very low loading $(<0.30)$.

For the ESEM, the fit indices indicated a substantially better fit of the data. Again, the extended seven-factor model was comparably better with RMSEA = 0.31 (CI90\% 0.029-0.033), CFI $=0.948$, TLI $=0.936$ and SRMR $=0.034$. However, all of the ESEM analyses indicated substantial cross-loadings of items $(<0.30)$ between several factors. Most notably, cross-loadings were substantial between "past negative" and "future negative", and between "past positive"/"future negative" and "present fatalistic". This clearly indicates that these factors were not differentiable from each other. Overall, considering the poor model fit and some high 


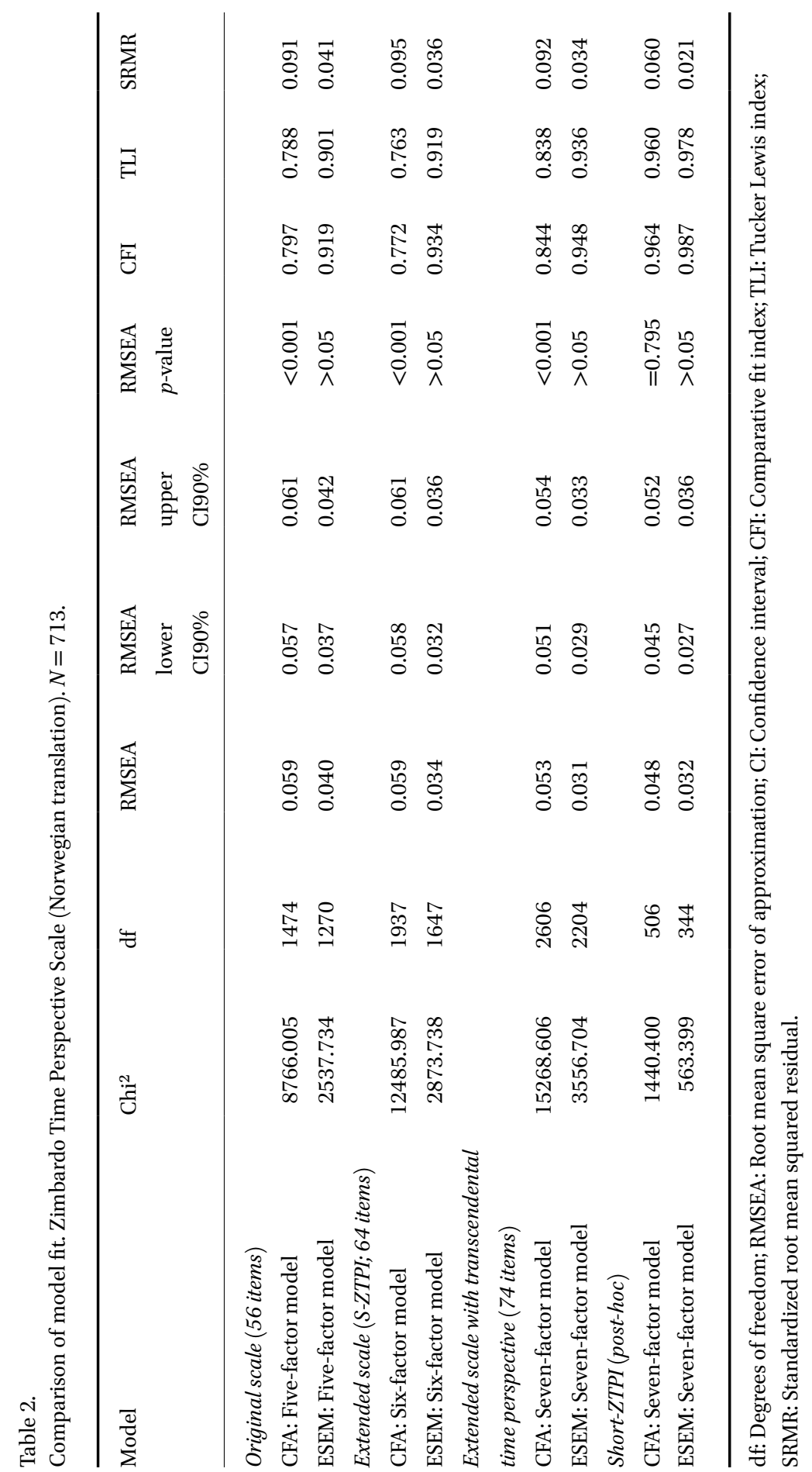


correlations between factors in the CFA, as well as substantial cross-loadings in the ESEM, none of the models included in the main analyses fitted the data.

\subsection{Post-Hoc Exploratory Analyses}

The exploratory factor analysis indicated that each of the suggested seven factors were uni-dimensional. A total of 27 items were, however, removed from further analyses due to low factor loadings. ESEM analyses indicated several items with substantial cross-loadings and some items with low loadings on all factors. Based on this, 13 more items were removed. Confirmatory factor analysis using the retained 34 items was subsequently done separately for each suggested factor. Model fit was adequate for all factors independently (RMSEA, range: 0.40-0.62; CFI, range: 0.934-0.998; TLI, range: 0.904-0.997; SRMR, range: 0.054-0.020).

Finally, a CFA was performed for the seven-factor model using the 34 retained items. This model had a RMSEA $=0.048$ (CI90\% 0.045-0.052), a CFI $=0.964$, $\mathrm{TIL}=0.960$ and SRMR $=0.060$, indicating an adequate to good fit with the data. The corresponding ESEM analysis also indicated appropriate fit (RMSEA $=0.032$ [CI90\% 0.027-0.036], CFI $=0.987$, TIL $=0.978$, SRMR $=0.021$ ). Cronbach's alpha for the factors ranged between 0.64 (future positive) and 0.89 (past negative), with a mean reliability of 0.76 across factors. The correlations between the factors ranged from 0.03 (present hedonistic-past positive) to 0.83 (present fatalisticfuture negative). In relation to measurement invariance, the 34-item version was found to be both configural and scalar invariant across gender $(\Delta$ between baseline and scalar model for gender: $\mathrm{RMSEA}=0.001, \mathrm{CFI}=0.008)$ and age $(\triangle \mathrm{RMSEA}$ $=0.003, \Delta \mathrm{CFI}=0.008$ ).

However, both the CFA and ESEM indicated poor discrimination between the future negative factor and the present fatalistic factor as indicated by high crossloadings (ESEM) and high factor correlations (CFA) in the 34-item model. Allowing for a higher-order factor mitigated this issue while still achieving an acceptable fit in CFA (RMSEA $=0.051$ [CI90\% 0.048-0.054]; CFI $=0.960$; TLI $=0.956$; SRMR $=0.064)$.

\section{Discussion}

\subsection{Summary of Findings}

The present study is the first attempt to compare different factor structures of the Norwegian version of ZTPI. For the original 56-item and the extended S-ZTPI version, our results are similar to those previously described (Carelli et al., 2011). The model that incorporated the transcendental time-perspective fitted the data somewhat better than the six-factor S-ZTPI version. However, none of the models tested initially fitted the data adequately. Even though CFI and TLI do 'not function well with correctly specified models that include a large number of variables' 
(Kenny \& McCoach, 2003, p. 349) and indications that commonly used cut-offs for fit indices are too restrictive (Beauducel \& Wittmann, 2005) especially for complex models (Marsh et al., 2004), there were also other problems with the models in our main analyses. These included high correlations between specific factors, several items with a low or very low factor loading, and a low internal reliability of the future positive factor. We therefore decided to perform an exploratory post-hoc analysis in an attempt to identify a model that fitted the data better. Following several data-driven steps, a model with 34 items and seven factors that had an acceptable to good fit was identified. For this model, the internal reliability of the different factors was high to very high, with the exception of the future positive time perspective.

\subsection{Implications}

Previous attempts have been made to shorten the ZTPI-inventory. For instance, 25-item (Wakefield et al., 2010), 20-item (Przepiorka et al., 2016), 18-item (Koštál et al., 2015), 17-item (Orosz et al., 2017) and 15-item (Koštál et al., 2015; McKay et al., 2014; Zhang et al., 2013) versions have been suggested. Although they were found to be psychometrically sound in the initial research reports, findings have been difficult to replicate (McKay et al., 2014, 2015; Worrell et al., 2018). For instance, in a Norwegian sample using factors from the 25-item version, it was necessary to remove one item to improve model fit for the purposes of a SEM-analysis (Pozolotina \& Olsen, 2018). Furthermore, the development of these short versions has been criticized for being too dependent on a data-driven approach as opposed to being theory-based (Temple et al., 2017; Worrell et al., 2018). Using a data-driven approach also increases the risk of over-fitting and poor external validity. We were not able to use the frequently advocated split-half approach due to lack of statistical power in our sample (Anderson \& Gerbing, 1988; Osborne \& Fitzpatrick, 2012). The findings from our post-hoc analysis should therefore be interpreted with caution, and we strongly encourage future research into the factor structure of ZTPI.

\subsection{Strengths and Limitations}

The main strength of the present study is the large sample size, which allowed for an initial investigation of the Norwegian version of the ZTPI. The main limitations relate to the inclusion of participants, which was based on a convenience approach, and the very low participation rate (9.1\%), as well the gender imbalance, where almost three quarters were female. The invitation was a mass e-mail to employees without individual tailoring, therefore the incentive to participate was most likely particularly low. The restricted sampling frame and participation rate curtails the potential for generalising the present findings to the Norwegian population per se. Furthermore, given the limited range of other variables included in the study, we could not investigate the convergent or divergent validity of ZTPI, 
nor investigate potential measurement non-invariance across other potentially important dimensions (such as educational level). Similarly, the high proportion of female participants may have restricted our ability to assess measurement invariance across gender. Finally, since the data was collected only once, we could not investigate the test-retest reliability of the Norwegian version of ZTPI.

\section{Conclusions}

This study did not find support for the original 56-item scale, for the S-ZTPI version, nor for an extended version which also incorporates the transcendental time perspective. In post-hoc analyses, we identified a model with 34 items and seven factors that fitted the data adequately. Our findings needs replication, and further studies should investigate the factor structure of ZTPI in a Norwegian context. International studies should investigate how the transcendental time perspective relates to the rest of ZTPI, while also exploring novel approaches to determine the best factor structure of the inventory.

\section{Acknowledgements}

We thank the study participants and the International Research Institute of Stavanger (IRIS) for the collection of the data and making them available for this study.

\section{Author Contributions}

The second author conceived the project, initiated and followed up the data collection. The first author carried out the literature review for the introduction and discussion sections, wrote the first draft of the manuscript, and performed the initial data analyses. The second author was involved in the preparation and planning of the statistical analyses, and both have reviewed and contributed to all parts of the written manuscript.

\section{Conflict of Interest Statement}

The authors declare that the research was conducted in the absence of any commercial or financial relationships that could be construed as a potential conflict of interest.

\section{References}

Akirmak, U. (2019). The validity and reliability of Zimbardo time perspective inventory in a Turkish sample. Curr. Psychol., ePub ahead of print. https://doi.org/10.1007/s12144-019-0153-3.

Anderson, J., \& Gerbing, D. (1988). Structural equation modelling in practice: A review and recommended two-step approach. Psychol. Bull., 103, 411-423.

Asparouhov, T., \& Muthén, B. (2009). Exploratory structural equation modeling. Struct. Equ. Modeling, 16, 397-438. 
Beauducel, A., \& Wittmann, W. W. (2005). Simulation study on fit indexes in cfa based on data with slightly distorted simple structure. Struct. Equ. Modeling, 12, 41-75.

Boyd, J. N., \& Zimbardo, P. G. (1997). Constructing time after death: The transcendental-future time perspective. Time Soc., 6, 35-54.

Boyd, J. N., \& Zimbardo, P. G. (2005). Time perspective, health, and risk taking. In A. Strathman \& J. Joireman (Eds), Understanding behavior in the context of time: Theory, research, and application. (pp. 85-107). Mahwah, NJ, USA: Lawrence Erlbaum Associates Publishers.

Byrne, B. M. (2012). Structural equation modeling with Mplus: Basic concepts, applications, and programming. New York, NY, SA: Routledge.

Carelli, M. G., Wiberg, B., \& Wiberg, M. (2011). Development and construct validation of the Swedish Zimbardo Time Perspective Inventory. Eur. J. Psychol. Assess., 27, 220-227.

Chan, S. M., Kwok, W. W., \& Fung, T. W. T. (2016). Psychometric properties of the Zimbardo time perspective inventory in Hong Kong adolescents. Time Soc., 28, 33-49.

Di Lernia, D., Serino, S., Pezzulo, G., Pedroli, E., Cipresso, P., \& Riva, G. (2018). Feel the time. Time perception as a function of interoceptive processing. Front. Hum. Neurosci., 12, 74. doi: 10.3389/ fnhum.2018.00074.

Enders, C. (2010). Applied missing data analysis. New York, NY, USA: Guilford.

Hair, J., Anderson, R., Tatham, R., \& Black, W. (1998). Factor analysis. In J. Hair, R. Anderson, R. Tatham, \& W. Black (Eds), Multivariate Data Analysis (5th ed) (pp. 87-140). Harlow, Essex, UK: Pearson Education Limited.

Hamilton, J. M., Kives, K. D., Micevski, V., \& Grace, S. L. (2003). Time perspective and health-promoting behavior in a cardiac rehabilitation population. Behav. Med., 28, 132-139.

Hu, L. T., \& Bentler, P. M. (1999). Cutoff criteria for fit indexes in covariance structure analysis: Conventional criteria versus new alternatives. Struct. Equ. Modeling, 6, 1-55.

Kenny, D. A., \& McCoach, D. B. (2003). Effect of the number of variables on measures of fit in structural equation modeling. Struct. Equ. Modeling, 10, 333-351.

Keough, K. A., Zimbardo, P. G., \& Boyd, J. N. (1999). Who's smoking, drinking, and using drugs? Time perspective as a predictor of substance use. Basic Appl. Soc. Psych., 21, 149-164.

Koštál, J., Klicperová-Baker, M., Lukavská, K., \& Lukavský, J. (2015). Short version of the Zimbardo Time Perspective Inventory (ZTPI-short) with and without the Future-Negative scale, verified on nationally representative samples. Time Soc., 25, 169-192.

Loose, T. T., Acier, D., Andretta, J. R., Cole, J. C., McKay, M. T., Wagner, V., \& Worrell, F. C. (2018). Time perspective and alcohol-use indicators in France and the United Kingdom: results across adolescents, university students, and treatment outpatients. Addict. Res. Theory, 26, 143-150.

Marsh, H. W., Hau, K.-T., \& Wen, Z. (2004). In search of golden rules: comment on hypothesis-testing approaches to setting cutoff values for fit indexes and dangers in overgeneralizing $\mathrm{Hu}$ and Bentler's (1999) findings. Struct. Equ. Modeling, 11, 320-341.

Marsh, H., Morin, A., Parker, P., \& Kaur, G. (2014). Exploratory structural equation modeling: An integration of the best features of exploratory and confirmatory factor analysis. Annu. Rev. Clin. Psychol., 10, 85-110. doi:10.1146/annurev-clinpsy-032813-153700.

McKay, M., Worrell, F. C., Temple, E. C., Perry, J., \& Cole, J. C. (2014). A critical examination of the reliability and validity of the SZTPI-15 in British, American and Australian samples. Pers. Individ. Dif., 69, 168-172.

McKay, M., Worrell, F., Temple, E., Perry, J., Cole, J., \& Mello, Z. (2015). Less is not always more: The case of the 36-item short form of the Zimbardo Time Perspective Inventory. Pers. Individ. Dif., $72,68-71$. 
Muthén, L. K., \& Muthén, B. (1998-2017). Mplus user's guide (8th ed.) Los Angeles, CA, USA: Muthén \& Muthén.

Orosz, G., Dombi, E., Tóth-Király, I., \& Roland-Lévy, C. (2017). The less is more: The 17-item Zimbardo Time Perspective Inventory. Curr. Psychol., 36, 39-47.

Osborne, J., \& Fitzpatrick, D. (2012). Replication analysis in exploratory factor analysis: What it is and why it makes your analysis better. Pract. Assess. Res. Eval., 17, 1-8.

Pozolotina, T., \& Olsen, S. O. (2018). Individual differences in time perspective, age, and smoking behavior: A test of two present versus future conceptualizations. J. Subst. Use, 23, 187-192.

Preau, M., Apostolidis, T., Francois, C., Raffi, F., \& Spire, B. (2007). Time perspective and quality of life among HIV-infected patients in the context of HAART. AIDS Care, 19, 449-458.

Przepiorka, A., Sobol-Kwapinska, M., \& Jankowski, T. (2016). A Polish short version of the Zimbardo Time Perspective Inventory. Pers. Indiv. Dif., 101, 78-89.

Putnick, D. L., \& Bornstein, M. H. (2016). Measurement invariance conventions and reporting: The state of the art and future directions for psychological research. Dev. Rev., 41, 71-90.

R Core Team (2018). R: A language and environment for statistical computing. Vienna, Austria: R Foundation for Statistical Computing. Retrieved from http://www.R-project.org/.

Rohr, M. K., John, D. T., Fung, H. H., \& Lang, F. R. (2017). A three-component model of future time perspective across adulthood. Psychol. Aging, 32, 597-607.

Rosellini, A. J., \& Brown, T. A. (2011). The NEO five-factor inventory: Latent structure and relationships with dimensions of anxiety and depressive disorders in a large clinical sample. Assessment, $18,27-38$.

Rosseel, Y. (2012). lavaan: An R package for structural equation modeling. J. Stat. Softw., 48, 1-36.

Sircova, A., van de Vijver, F. J. R., Osin, E., Milfont, T. L., Fieulaine, N., Kislali-Erginbilgic, A., .. . Boyd, J. N. (2014). A Global Look at Time: A 24-country study of the equivalence of the Zimbardo Time Perspective Inventory. SAGE Open, 4, 2158244013515686. doi:10.1177/2158244013515686.

Sobol-Kwapinska, M., Przepiorka, A., \& Zimbardo, P. P. (2016). The structure of time perspective: Age-related differences in Poland. Time Soc., 28, 5-32.

Sobol-Kwapinska, M., Jankowski, T., Przepiorka, A., Oinyshi, I., Sorokowski, P., \& Zimbardo, P. (2018). What is the structure of time? A study on time perspective in the USA, Poland, and Nigeria. Front. Psychol. 9, 2018.02078.doi: 10.3389/fpsyg.2018.02078.

Stolarski, M., Fieulaine, N., \& van Beek, W. (2015). Time Perspective Theory: Review, research and application. New York, NY, USA: Springer.

Stolarski, M., Fieulaine, N., \& Zimbardo, P. G. (2018). Putting time in a wider perspective: The past, the present, and the future of time perspective theory. In V. Zeigler-Hill \& T. K. Shackelford (Eds), The SAGE handbook of personality and individual differences (592-628). Thousand Oakes, CA, USA: SAGE.

Temple, E., Perry, J. L., Worrell, F. C., Zivkovic, U., Mello, Z. R., Musil, B., .. McKay, M. T. (2017). The Zimbardo time perspective inventory: Time for a new strategy, not more new shortened versions. Time Soc., 0961463X17718102. doi: 10.1177/0961463X17718102.

Wakefield, C. E., Homewood, J., Taylor, A., Mahmut, M., \& Meiser, B. (2010). Time perspective in hereditary cancer: Psychometric properties of a short form of the Zimbardo Time Perspective Inventory in a community and clinical sample. Genet. Test Mol. Biomarkers, 14, 617-627.

Worrell, F. C., Temple, E. C., McKay, M. T., Živkovič, U., Perry, J. L., Mello, Z. R., . . Cole, J. C. (2018). A theoretical approach to resolving the psychometric problems associated with the Zimbardo Time Perspective Inventory: Results from the USA, Australia, Slovenia, and the United Kingdom. Eur. J. Psych. Assess., 34, 41-51. 
Zhang, J. W., Howell, R. T., \& Bowerman, T. (2013). Validating a brief measure of the Zimbardo Time Perspective Inventory. Time Soc., 22, 391-409.

Zimbardo, P. G., \& Boyd, J. N. (1999). Putting time in perspective: A valid, reliable individual-differences metric.J. Pers. Soc. Psychol., 77, 1271-1288.

Zimbardo, P. G., Keough, K. A., \& Boyd, J. N. (1997). Present time perspective as a predictor of risky driving. Pers. Indiv. Dif., 23, 1007-1023. 